\title{
Comparative study of Gamma Knife surgery and percutaneous retrogasserian glycerol rhizotomy for trigeminal neuralgia in patients with multiple sclerosis
}

\author{
Clinical article
}

\author{
David Mathieu, M.D., F.R.C.S.C., Khaled EfFendi, M.D., \\ Jocelyn Blanchard, M.D., F.R.C.S.C., ANd Mario Séguin, M.D., F.R.C.S.C.
}

Division of Neurosurgery, Department of Surgery, Université de Sherbrooke, Centre Hospitalier Universitaire de Sherbrooke, Sherbrooke, Quebec, Canada

\begin{abstract}
Object. Among patients with multiple sclerosis (MS) there is a high incidence of trigeminal neuralgia (TN), and outcomes after treatment seem inferior to those in patients suffering from idiopathic TN. The goal of this study was to evaluate clinical outcomes in patients with MS-related TN after Gamma Knife surgery (GKS) and compare them with those obtained using percutaneous retrogasserian glycerol rhizotomy (PRGR).

Methods. The authors retrospectively reviewed the charts of 45 patients with MS-related TN. The first procedure undertaken was GKS in 27 patients and PRGR in 18 patients. Pain had been present for a median of 60 months (range 12-276 months) in patients who underwent GKS and 48 months (range 12-240 months) in patients who underwent PRGR. The following outcome measures were assessed in both groups of patients: pain relief (using the Barrow Neurological Institute [BNI] Pain Scale), procedure-related morbidity, time to pain relief and recurrence, and subsequent procedures that were performed.

Results. The median duration of follow-up was 39 months (range 13-69 months) in the GKS group and 38 months (range 2-75 months) in the PRGR group. Reasonable pain control (BNI Pain Scale Scores I-IIIb) was noted in 22 patients $(81.5 \%)$ who underwent GKS and in 18 patients $(100 \%)$ who underwent PRGR. For patients who underwent GKS, the median time to pain relief was 6 months; for those who underwent PRGR, pain relief was immediate. In the GKS group12 patients required subsequent procedures (3 patients for absence of response and 9 patients for pain recurrence), whereas in the PRGR group 6 patients required subsequent procedures (all for pain recurrence). As of the last follow-up, complete or reasonable pain control was finally achieved in 23 patients $(85.2 \%)$ in the GKS group and in 16 patients $(88.9 \%$ ) in the PRGR group. The morbidity rate was $22.2 \%$ in the GKS group (all due to sensory loss and paresthesia) and $66.7 \%$ in the PRGR group (mostly hypalgesia, with 2 patients having corneal reflex loss and 1 patient suffering from meningitis).

Conclusions. Both GKS and PRGR are satisfactory strategies for treating MS-related TN. Gamma Knife surgery has a lower rate of sensory and overall morbidity than PRGR, but requires a delay before pain relief occurs. The authors propose that patients with extreme pain in need of fast relief should undergo PRGR. For other patients, both management strategies can lead to satisfactory pain relief, and the choice should be made based on patient preference and expectations.
\end{abstract}

(http://thejns.org/doi/abs/10.3171/2012.6.GKS12987)

KEY WORDS • trigeminal neuralgia $\bullet$ multiple sclerosis $\bullet$
Gamma Knife surgery $\bullet \quad$ percutaneous retrogasserian glycerol rhizotomy
stereotactic radiosurgery

$\mathrm{M}$ ULTIPLE sclerosis is an inflammatory disease that causes central nervous system demyelination. This common disease has a prevalence of $2-150$ per 100,000 population and is most frequently found in white people. Trigeminal neuralgia occurs in approximately $2 \%$ of patients with MS, a percentage 20 times higher than that in the general population. ${ }^{4}$ Bilateral pain will develop in approximately $11 \%-32 \%$ of patients with

Abbreviations used in this paper: $\mathrm{BNI}=$ Barrow Neurological Institute; GKS = Gamma Knife surgery; MS = multiple sclerosis; PRGR = percutaneous retrogasserian glycerol rhizotomy; $\mathrm{TN}=$ trigeminal neuralgia.
MS-related TN. ${ }^{4}$ In this setting TN develops as a result of demyelinating plaques that occur in the central trigeminal pathways or the proximal part of the trigeminal nerve. ${ }^{2,9}$ Unlike idiopathic TN, vascular compression of the root entry zone does not seem to contribute to the disease in patients with MS, and as such, microvascular decompression confers no significant benefit to this population. ${ }^{12} \mathrm{As}$ in idiopathic cases, medical management using carbamazepine or related drugs represents the first line of treatment for TN in patients with MS; however, in these patients the pain is often more difficult to relieve and does not seem to respond as well. ${ }^{3}$ In cases of medically refractory pain, percutaneous rhizotomy can be performed 
with good reported rates of pain relief. . $3,7,10$ In patients with idiopathic TN, GKS reportedly produces excellent pain relief ${ }^{6,8,13,15}$ that is comparable to the relief obtained with percutaneous procedures; however, fewer successful cases have been reported in patients with MS. ${ }^{16}$

The goal of this study was to compare outcomes following GKS and PRGR in patients with MS who were treated for TN at our institution.

\section{Methods}

\section{Data Collection and Analyses}

This retrospective study was approved by the local institutional ethics committee. Patients were included in the study if they had undergone their first intervention for MS-related TN (either GKS or PRGR) at our institution between January 2004 and December 2009. All patients had proven MS based on clinical and radiological criteria. Four neurosurgeons were involved in the care of the patients, and treatment was chosen according to patient and physician preference as well as the severity of the patient's pain. Glycerol rhizotomy is the only percutaneous procedure performed at our institution. In general, patients with severe acute pain were offered PRGR. Patients experiencing V1 pain were treated with GKS, because of the relative difficulty in affecting V1 sensation with PRGR. Data were collected by a physician who was not directly involved in the patients' care. The study data included patient demographics, technical procedural details, pain relief outcomes, and treatment-related morbidity; these were obtained by reviewing patient medical records and by phone interviews with patients. Pain outcome was rated using the BNI Pain Scale, in which the following scores apply: I, no trigeminal pain with no medication; II, occasional pain that requires no medication; IIIa, no pain and medication is used; IIIb some pain, which is adequately controlled using medication; IV, some pain, which is not adequately controlled using medication; and $\mathrm{V}$, severe pain that has no relief even with medication. ${ }^{14}$ Statistical analyses were performed using commercially available software (SPSS version 16.0, SPSS Inc.).

\section{Gamma Knife Surgery}

Twenty-seven patients underwent GKS as their first procedure. The median age of these patients at GKS was 59 years (range $42-78$ years); 15 patients were female and 12 were male. These patients had suffered from TN for a median of 60 months (range 12-276 months). Fifteen patients had pain on the right side and 12 had pain on the left side. The most commonly affected nerve roots were $\mathrm{V} 2$ and V3. The pain was considered typical in 13 patients (paroxysmal electric-shock pain) and atypical in 14 (constant pain with or without superimposed paroxysmal episodes). Table 1 displays a comparison of demographic and clinical data in both groups of patients.

After consent had been obtained for the procedure, patients received local anesthesia with conscious sedation, and a Leksell G stereotactic frame (Elekta Instruments $A B$ ) was installed on the head. Patients were then transported to the MR imaging suite so that we could

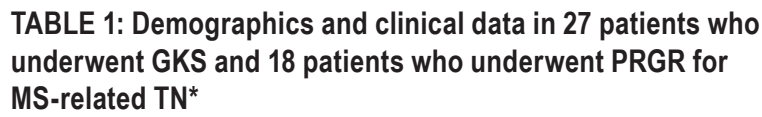

\begin{tabular}{lcc}
\hline \multicolumn{1}{c}{ Variable } & GKS Cases & PRGR Cases \\
\hline sex & & \\
female & $15(55.6)$ & $9(50)$ \\
male & $12(44.4)$ & $9(50)$ \\
median age in yrs (range) & $59(42-78)$ & $56.5(41-79)$ \\
median duration of symptoms in mos & $60(12-276)$ & $48(12-240)$ \\
$\quad$ (range) & & \\
side of pain & & \\
rt & $15(55.6)$ & $9(50)$ \\
It & $12(44.4)$ & $9(50)$ \\
distribution of pain & & \\
V2 \& V3 & $11(40.7)$ & $9(50)$ \\
V3 & $10(37)$ & $8(44.4)$ \\
V2 & $3(11.1)$ & $1(5.6)$ \\
V1, V2, \& V3 & $2(7.4)$ & 0 \\
V1 \& V3 & $1(3.7)$ & 0 \\
trigger zone & & \\
yes & $24(88.9)$ & $16(88.9)$ \\
no & $3(11.1)$ & $2(11.1)$ \\
type of pain & $14(51.9)$ & $11(61.1)$ \\
atypical & $13(48.1)$ & $7(38.9)$ \\
typical & & \\
\hline
\end{tabular}

* Values are presented as the number of patients (\%) unless otherwise noted.

obtain a volumetric contrast-enhanced magnetizationprepared rapid acquisition with gradient echo sequence, which was used for dosimetry planning. A CT myelogram was obtained in 2 patients in whom MR imaging was contraindicated. The trigeminal nerve was targeted in its cisternal segment by using a single 4-mm isocenter. A maximum radiation dose of 80-90 Gy was delivered to the nerve. The isocenter was placed as close as possible to the root entry zone, with the goal of keeping the dose to the brainstem lower than $20 \mathrm{~Gy}$. Nineteen patients were treated with a dose of $80 \mathrm{~Gy}, 7$ patients with $85 \mathrm{~Gy}$, and 1 patient with $90 \mathrm{~Gy}$. In two-thirds of patients there was clear MR evidence of pons demyelination, and interestingly, in 2 patients there was evidence of trigeminal vascular compression. All patients were treated by using a Leksell Gamma Knife model 4C (Elekta Instruments AB). After the procedure, the patients were observed for 30 minutes and then discharged on the same day. Details of the radiosurgery procedure are shown in Table 2.

\section{Glycerol Rhizotomy}

Eighteen patients underwent PRGR as their first procedure (Table 1). The median age in this group of patients was 56.5 years (range $41-79$ years); 9 patients were male and 9 were female. The patients had suffered from TN for a median of 48 months (range 12-240 months). Again, V2 and V3 were most commonly involved, and the TN was 
TABLE 2: Details of GKS in 27 patients treated for MS-related TN

\begin{tabular}{cc}
\hline \multicolumn{1}{c}{ Variable } & No. of Patients (\%) \\
\hline imaging modality & $25(92.6)$ \\
MRI & $2(7.4)$ \\
CT myelography & \\
imaging findings & $18(66.7)$ \\
pons demyelination & $2(7.4)$ \\
vascular compression & \\
radiation dose (Gy) & $19(70.4)$ \\
80 & $7(25.9)$ \\
85 & $1(3.7)$ \\
90 &
\end{tabular}

equally distributed between the right and left sides. Typical pain was present in 7 patients and atypical pain in 11 patients. All patients underwent MR imaging before the procedure, and plaque was seen in the pons in 16 patients.

The patients were transported to the operating room. The procedure was conducted using local anesthesia with conscious sedation, and constant cardiorespiratory monitoring was performed by the anesthesiologist. Patients were placed supine. With the aid of fluoroscopic guidance, a 22-gauge spinal needle was inserted through the foramen ovale using standard cutaneous landmarks. Upon entering the foramen, mild jaw contraction and pain reproduction were expected. Egress of cerebrospinal fluid from the needle could be seen in 13 patients. The patients were then placed in the sitting position, and cisternography was performed using iohexol dye (Omnipaque, GE Healthcare). The volume of the Meckel cave was noted. After the dye had been washed out, we slowly injected 99\% pure glycerol while again noting pain reproduction and facial paresthesia. Bradycardia was observed in 5 patients during the injection. The total volume of injected glycerol was based on the volume of the Meckel cave (plus $0.1 \mathrm{ml}$ to account for dead space in the needle lumen) and the appearance of sensory symptoms. The volume of glycerol varied between 0.4 and $1.0 \mathrm{ml}$, with most patients receiving $0.6 \mathrm{ml}$ or less. Afterward, the needle was withdrawn, and the patients were kept in the sitting position for 2 hours. Patients were discharged from the hospital on the following day. Table 3 reports the operative details in patients who underwent PRGR.

\section{Results}

\section{Gamma Knife Surgery}

The median follow-up time after GKS was 39 months (range 13-69 months). Satisfactory pain relief (BNI Pain Scale Score I-IIIb) was achieved in 22 patients (81.4\%). The median time to optimal pain relief was 6 months (range 0.5-13 months). Fourteen patients (51.9\%) eventually experienced pain recurrence; the median time to recurrence was 26.5 months. According to Cox regression analyses, treatment doses higher than 80 Gy were associated with an increased likelihood of pain relief ( $\mathrm{p}$ $=0.003)$ and lower rates of pain recurrence $(p=0.002)$.
TABLE 3: Operative details in 18 patients who underwent PRGR for MS-related TN

\begin{tabular}{cc}
\hline Variable & No. of Patients $(\%)$ \\
\hline surgical findings & \\
bradycardia & $5(27.8)$ \\
trismus & $4(22.2)$ \\
CSF flow & $13(72.2)$ \\
pain reproduction & $16(88.9)$ \\
glycerol (ml) & \\
0.4 & $1(5.6)$ \\
0.45 & $8(44.4)$ \\
0.5 & $1(5.6)$ \\
0.6 & $3(16.7)$ \\
0.7 & $2(11.1)$ \\
0.8 & $2(11.1)$ \\
1 & $1(5.6)$ \\
\hline
\end{tabular}

The presence of pontine plaque (detected on MR images) or atypical pain had no impact on treatment outcomes.

Twelve patients underwent additional procedures (GKS in 2 patients and PRGR in 10 patients) for unsatisfactory initial relief (3 patients) or pain recurrence $(9$ patients). Five patients declined additional procedures. At the time of the last follow-up examination, 23 patients (85.2\%) reported satisfactory pain control. Table 4 displays comparative pain relief rates between the GKS and PRGR groups. Figure 1 details the final outcomes in patients who underwent GKS.

After GKS, 6 patients (22.2\%) reported some form of new trigeminal neuropathy. According to the BNI Facial Numbness Scale, ${ }^{14}$ this was Score 2 (mild and not bothersome) in 4 patients and Score 3 (moderate, somewhat bothersome) in 2 patients. No patient reported severe and very bothersome numbness related to the procedure.

\section{Percutaneous Glycerol Rhizotomy}

The median follow-up time after PRGR was 38 months (range 2-75 months). Every patient (100\%) experienced immediate ( $\leq 24$ hours after the procedure) pain relief (BNI Pain Scale Score I in 8 patients and IIIa in 10 patients) (Table 4). Seven patients (38.9\%) eventually experienced pain recurrence, at a median of 17 months. Ac-

TABLE 4: Pain outcomes after GKRS and PRGR*

\begin{tabular}{cccccc}
\hline & \multicolumn{2}{c}{ GKS Group } & & \multicolumn{2}{c}{ PRGR Group } \\
\cline { 2 - 3 } \cline { 6 - 6 } BNI Facial Pain Score & Initial & Final & & Initial & Final \\
\hline I II & $7(25.9)$ & $4(14.8)$ & & $8(44.4)$ & $6(33.3)$ \\
IIla & $1(3.7)$ & $1(3.7)$ & & $0(0)$ & $0(0)$ \\
IIIb & $2(44.4)$ & $14(51.9)$ & & $10(55.6)$ & $9(50)$ \\
IV & $2(7.4)$ & $4(14.8)$ & & $0(0)$ & $1(5.6)$ \\
V & $3(11.1)$ & $1(3.7)$ & & $0(0)$ & $1(5.6)$ \\
\hline
\end{tabular}

* Values are presented as the number of patients (\%). 


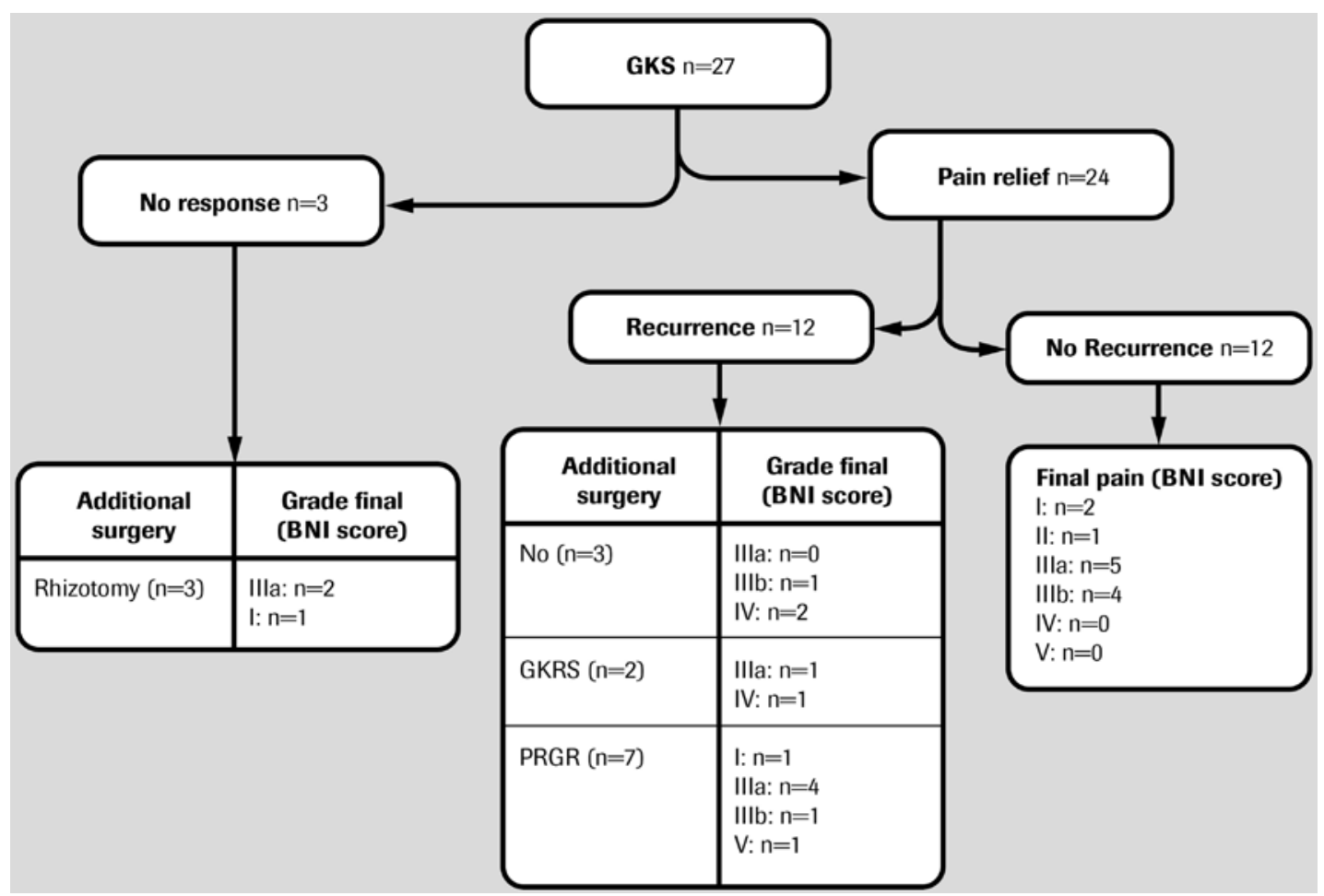

FIG. 1. Summary of patient outcomes after GKS (GKRS).

cording to Cox regression analyses, only the presence of trismus at surgery correlated to better initial pain relief ( $\mathrm{p}$ $=0.019)$ and a lower likelihood of recurrence $(p=0.016)$. No other surgical finding, MR imaging finding, or type of pain had any impact on outcome.

Six patients required additional surgery for pain recurrence (repeated PRGR in 5 patients and repeated PRGR and GKS in 1 patient). One patient declined further surgery. Figure 2 details outcomes of patients in the PRGR group. Overall, satisfactory pain relief was achieved in 16 patients $(88.9 \%)$ as of the last follow-up examination.

Morbidity from the procedure occurred in 12 patients $(66.7 \%)$. Sensory side effects were experienced by 11 patients $(61.1 \%)$ after PRGR. These were reported as BNI Facial Numbness Scale Score 2 by all of these patients. In 2 of the patients the corneal reflex on the operated side was abolished. One patient (5.6\%) suffered from bacterial meningitis after PRGR; this resolved without complication after appropriate antibiotic treatment. The causal organism was Streptococcus viridans, and the illness was probably caused by an inadvertent and unrecognized intraoral mucosal breach during the procedure.

\section{Discussion}

As previously stated, TN occurs with greater frequency in patients with MS and is often more difficult to treat in them than it is in the general population. Gamma Knife surgery has been reported to provide pain relief in $60 \%-80 \%$ of treated patients in several large series; $;, 8,13,15$ MS has been reported to be a negative predictive factor in most cases. In a review of 365 patients treated by GKS for TN by Verheul et al., ${ }^{16} 31$ patients had MS. Only $46 \%$ of patients with MS obtained adequate pain relief at 5 years, compared with $75 \%$ of patients with idiopathic TN. Articles focusing on GKS for MS-related TN are few in the literature. Huang et al. ${ }^{5}$ reported outcomes in 7 patients with MS who underwent GKS for TN. All of the patients became pain free after a delay of 1 day to 8 months. Four patients $(57 \%)$ had numbness as a consequence of the treatment. Zorro and colleagues ${ }^{17}$ reported the University of Pittsburgh experience of 37 patients. They achieved complete pain relief in $62.1 \%$ of patients and reasonable pain control (BNI Pain Scale Scores I-IIIb) in 97.3\%, within 90 days of radiosurgery. Recurrent pain occurred in $37.8 \%$ of patients. Sensory morbidity was noted in $5.4 \%$ of patients. Our results after GKS are similar to those reported by Zorro and colleagues, although the time to pain relief was longer in our series. We have no explanation for this longer time, because our patient population and treatment parameters were similar to those in their study. It may be related to the underlying pathophysiology of the disease. A longer time to response was also noted by Huang et al. ${ }^{5}$ According to our findings and congruent with results in other series, the prognosis for pain relief after GKS in patients with MS appears similar to what is reported in idiopathic cases, although recurrence rates seem higher in patients with MS. ${ }^{3}$

Percutaneous rhizotomy has long been used to treat $\mathrm{TN}$ in patients with MS, because of the relative contraindication to microvascular decompression. Kondziolka et al. ${ }^{7}$ described 53 patients who underwent PRGR in this setting. Long-term complete pain relief was achieved in 


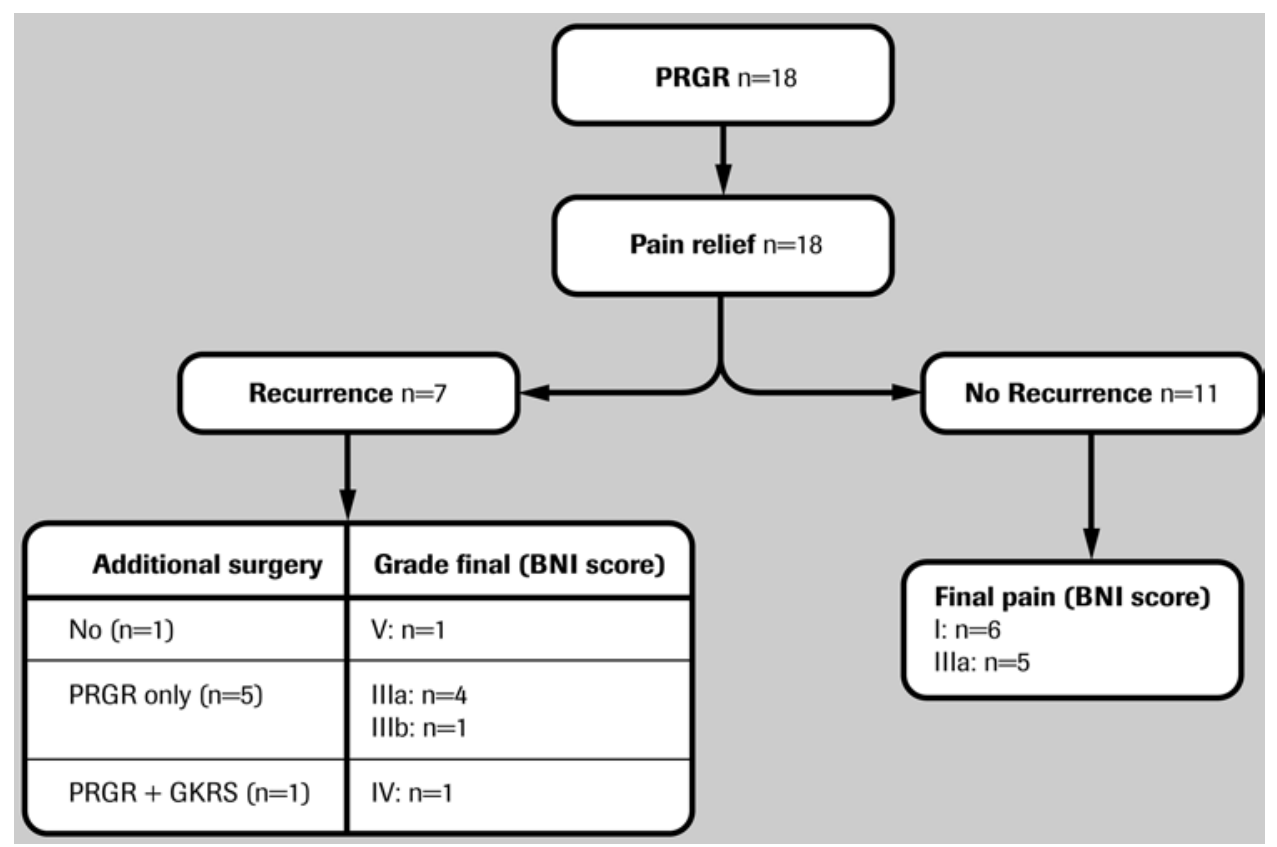

FIG. 2. Summary of patient outcomes after PRGR.

$59 \%$ of patients, and an additional $16 \%$ of patients only required occasional medication. Pickett et al. ${ }^{11}$ performed PRGR in 53 patients; immediate complete pain relief was obtained in $78 \%$ and partial relief in an additional $13 \%$. Pain recurrence was reported by $59 \%$ of patients. Twentyfour patients underwent repeated PRGR with pain control rates similar to those of the first procedure. In our experience, PRGR produced pain relief in all of our patients. At the last follow-up examination, $89 \%$ of patients had satisfactory pain control. This compares favorably with previously published results. We observed a significantly higher rate of permanent sensory changes in our series than rates reported in other papers. This is probably related to the high volume of injected glycerol used in our series.

Based on our results, it seems that both GKS and PRGR can be satisfying surgical solutions for patients with MS who suffer from intractable TN. Since those patients will likely require multiple procedures due to pain recurrence, we offer GKS as the first-line therapy to most patients. We do this because GKS is associated with lower rates of overall sensory morbidity and to avoid the long-term risk of deafferentiation pain. The majority of patients can tolerate the latency period before the treatment effect. For patients with excruciating pain that interferes with oral intake, PRGR should be the option of choice to afford fast relief. Both options need to be discussed with patients, however, emphasizing the risks and benefits and taking into account individual clinical features and patient choice.

Our study suffers from some limitations. First, our patient sample is small due to the relative rarity of the disease in this specific situation. Second, the retrospective nature of our study makes the study subject to a recall bias and limited to available data in the patient charts. Finally, the treatment allocation to groups was not ran- dom, but rather based on clinician and patient preference. Despite these limitations, we believe that our results are valid and will help physicians involved in the care of these difficult-to-treat patients. A prospective randomized study designed to compare GKS and PRGR in patients with MS would certainly be of value to strengthen these results.

\section{Conclusions}

Gamma Knife surgery and PRGR are both satisfactory treatment strategies for patients with MS who suffer from intractable TN. Most patients treated with GKS will obtain pain relief after a latency period, which can last many months. Glycerol rhizotomy achieves pain relief faster and more efficiently than GKS, but carries higher overall risks and sensory morbidity. We propose that patients with MS-related TN who are in acute pain undergo PRGR first to produce fast pain relief. For other patients, the choice of treatment should be made based on patient preference and expectations. In the long term, it is possible to achieve satisfactory pain relief for most patients independent of the original surgical strategy, by repetition of treatment or by switching to the other procedure as needed.

\section{Disclosure}

The authors report no conflict of interest regarding the materials or methods used in this study or the findings specified in this paper.

Author contributions to the study and manuscript preparation include the following. Conception and design: Mathieu, Séguin. Acquisition of data: Effendi. Analysis and interpretation of data: Mathieu, Effendi. Drafting the article: Mathieu. Critically revising the article: all authors. Reviewed submitted version of manuscript: all authors. Approved the final version of the manuscript on behalf of all authors: Mathieu. Administrative/technical/material support: Mathieu, Blanchard, Séguin. Study supervision: Mathieu. 


\section{References}

1. Brett DC, Ferguson GG, Ebers GC, Paty DW: Percutaneous trigeminal rhizotomy. Treatment of trigeminal neuralgia secondary to multiple sclerosis. Arch Neurol 39:219-221, 1982

2. Cruccu G, Biasiotta A, Di Rezze S, Fiorelli M, Galeotti F, Innocenti $\mathrm{P}$, et al: Trigeminal neuralgia and pain related to multiple sclerosis. Pain 143:186-191, 2009

3. De Santi L, Annunziata P: Symptomatic cranial neuralgias in multiple sclerosis: clinical features and treatment. Clin Neurol Neurosurg 114:101-107, 2012

4. Hooge JP, Redekop WK: Trigeminal neuralgia in multiple sclerosis. Neurology 45:1294-1296, 1995

5. Huang E, Teh BS, Zeck O, Woo SY, Lu HH, Chiu JK, et al: Gamma knife radiosurgery for treatment of trigeminal neuralgia in multiple sclerosis patients. Stereotact Funct Neurosurg 79:44-50, 2002

6. Knafo H, Kenny B, Mathieu D: Trigeminal neuralgia: outcomes after gamma knife radiosurgery. Can J Neurol Sci 36: 78-82, 2009

7. Kondziolka D, Lunsford LD, Bissonette DJ: Long-term results after glycerol rhizotomy for multiple sclerosis-related trigeminal neuralgia. Can J Neurol Sci 21:137-140, 1994

8. Kondziolka D, Perez B, Flickinger JC, Habeck M, Lunsford LD: Gamma knife radiosurgery for trigeminal neuralgia: results and expectations. Arch Neurol 55:1524-1529, 1998

9. Love S, Gradidge T, Coakham HB: Trigeminal neuralgia due to multiple sclerosis: ultrastructural findings in trigeminal rhizotomy specimens. Neuropathol Appl Neurobiol 27:238244, 2001

10. Montano N, Papacci F, Cioni B, Di Bonaventura R, Meglio M: Percutaneous balloon compression for the treatment of trigeminal neuralgia in patients with multiple sclerosis. Analysis of the potentially prognostic factors. Acta Neurochir (Wien) 154:779-783, 2012

11. Pickett GE, Bisnaire D, Ferguson GG: Percutaneous retrogasserian glycerol rhizotomy in the treatment of tic douloureux associated with multiple sclerosis. Neurosurgery 56:537-45, 2005

12. Resnick DK, Jannetta PJ, Lunsford LD, Bissonette DJ: Micro- vascular decompression for trigeminal neuralgia in patients with multiple sclerosis. Surg Neurol 46:358-362, 1996

13. Régis J, Metellus P, Hayashi M, Roussel P, Donnet A, BilleTurc F: Prospective controlled trial of gamma knife surgery for essential trigeminal neuralgia. J Neurosurg 104:913-924, 2006

14. Rogers CL, Shetter AG, Fiedler JA, Smith KA, Han PP, Speiser BL: Gamma knife radiosurgery for trigeminal neuralgia: the initial experience of The Barrow Neurological Institute. Int J Radiat Oncol Biol Phys 47:1013-1019, 2000

15. Sheehan JP, Ray DK, Monteith S, Yen CP, Lesnick J, Kersh R, et al: Gamma Knife radiosurgery for trigeminal neuralgia: the impact of magnetic resonance imaging-detected vascular impingement of the affected nerve. Clinical article. J Neurosurg 113:53-58, 2010

16. Verheul JB, Hanssens PEJ, Lie ST, Leenstra S, Piersma H, Beute GN: Gamma Knife surgery for trigeminal neuralgia: a review of 450 consecutive cases. Clinical article. J Neurosurg 113 Suppl:160-167, 2010

17. Zorro O, Lobato-Polo J, Kano H, Flickinger JC, Lunsford LD, Kondziolka D: Gamma knife radiosurgery for multiple sclerosis-related trigeminal neuralgia. Neurology 73:1149-1154, 2009

Manuscript submitted May 15, 2012.

Accepted June 11, 2012.

Portions of this work were presented orally at the 16th International Leksell Gamma Knife Society Meeting (Sydney, Australia, March 25-29, 2012) and at the Gamma Knife Radiosurgery in the Americas meeting of the North American Gamma Knife Consortium (Pittsburgh, Pennsylvania, September 17-19, 2011). This work was also presented in poster form at the Congress of Neurological Surgeons 2011 Annual Meeting (Washington, DC, October 1-6, 2011).

Please include this information when citing this paper: DOI: 10.3171/2012.6.GKS12987.

Address correspondence to: David Mathieu, M.D., F.R.C.S.C., Division of Neurosurgery, Centre Hospitalier Universitaire de Sherbrooke, 3001, 12e avenue Nord, Sherbrooke, Quebec J1H 5N4, Canada. email: david.mathieu@usherbrooke.ca. 